\title{
Recognition Algorithm for Diamond-Free Graphs
}

\author{
Mihai TALMACIU, Elena NECHITA \\ Department of Mathematics and Informatics, University of Bacău \\ 8 Spiru Haret str., 600114 Bacău, Romania \\ e-mail:mihaitalmaciu@yahoo.com,elenechita@yahoo.com
}

Received: May 2006

\begin{abstract}
In this paper we recall the notion of weakly decomposition, we recall some necessary and sufficient conditions for a graph to admit such a decomposition, we introduce the recognition algorithm for the diamond-free graphs which keeps the combinatorial structure of the graph by means of the decomposition, as well as an easy possibility to determine the clique number for the diamond-free graphs.
\end{abstract}

Key words: diamond graph, weakly decomposition, recognition algorithm.

\section{Introduction}

Let $G=(V, E)$ be a finite undirected graph without self-loops and multiple edges. In different problems in the theory of graphs, particularly in the building of some recognition algorithms, frequently appears a type of partition of the set of vertices in tree classes $A$, $B, C$ such that $A$ induces a connected graph, and $C$ is totally adjacent to $B$ and totally nonadjacent to $A$.

We recall some results concerning the diamond-free graphs, but which are also prime.

Theorem (see (Brandstadt, 2002)). If $G$ is a prime \{diamond, co-diamond\}-free graph then $G$ or $\bar{G}$ is a co-matched bipartite graph or has at most nine vertices.

(A graph $G$ is a matched co-bipartite graph if $G$ is partitionable into two cliques $C_{1}$, $C_{2}$ with $\left|C_{1}\right|=\left|C_{2}\right|$ or $\left|C_{1}\right|=\left|C_{2}\right|+1$ such that the edges between $C_{1}$ and $C_{2}$ determine a matching and at most one vertex in $C_{1}$ and $C_{2}$ is not covered by the matching. A graph $G$ is a co-matched bipartite graph if it is the complement of a matched co-bipartite graph).

Theorem (see (Brandstadt et al., 2003)). If $G$ is a prime $\left\{P_{5}\right.$, diamond $\}$-free graph then $G$ is a thin spider or $G$ is an enhanced bipartite chain graph or $G$ has at most nine vertices.

( $G$ is a thin spider if its vertices set is partitionable into a clique $C$ and a stable set $S$ with $|C|=|S|$ or $|C|=|S|+1$ such that the edges between $C$ and $S$ determine a matching and at most one vertex in $C$ is not covered by the matching. $G$ is an enhanced bipartite 
chain graph if it is the complement of an enhanced co-bipartite chain graph. $G$ is an enhanced co-bipartite chain graph if it is partitionable into a co-bipartite chain graph with clique $C_{1}, C_{2}$ and three additional vertices $a, b, c$ ( $a$ and $c$ optional) such that $N(a)=$ $C_{1} \cup C_{2}, N(b)=C_{1}$ and $N(c)=C_{2}$, and there are no other edges in $G, G$ is a cobipartite chain graph if it is the complement of a bipartite chain graph. A bipartite graph $B=(X, Y, E)$ is a bipartite chain graph if there is an ordering $x_{1}, x_{2}, \ldots, x_{n}$ of all vertices in $X$ such that $N\left(x_{i}\right) \subseteq N\left(x_{j}\right)$ for all $1 \leqslant i<j \leqslant k$ ).

Theorem (see (Brandstadt et al., 2003)). The classes of graphs \{diamond, co-diamond $\}$ free, $\{$ diamond, co-paw $\}$-free, $\left\{\right.$ diamond, $\left.2 K_{2}\right\}$-free, $\left\{K_{4}, c o-\right.$ diamond $\}$-free have bounded clique-width.

Theorem (see (Brandstadt et al., 2003)). The class of graphs $\left\{K_{4}\right.$, diamond, $C_{4}$, claw $\}$ free has unbounded clique-width.

Theorem (see (Kloks et al.)). There exists an $\mathrm{O}\left(\mathrm{e}^{3 / 2}+n^{\alpha}\right)$ algorithm that checks if a graph has a diamond and produces one if it does.

In (Brandstadt, 2002), A. Brandstadt gives the structure and linear time optimization of the ( $P_{5}$, diamond)-free graphs.

A question is arises: what properties have the diamond-free graphs, but which are not also prime.

\section{Notations and Fundamental Definitions}

Throughout this paper (see (Berge, 1985)) $G=(V, E)$ is a simple (i.e., finite, undirected, without loops and multiple edges) graph. Let $c o-G=\bar{G}$ denote the complement graph of $G$. For $U \subseteq V$ let $[U]$ denote the subgraph of $G$ induced by $U$. Throughout this paper, all subgraphs are understood to be induced subgraphs. By $G-X$ we mean the graph $[V-X]$, whenever $X \subseteq V$, but we shall often denote it simply $G-v(\forall v \in V)$ when there is no ambiguity. If $v \in V$ is a vertex in $G$, the neighborhood $N_{G}(v)$ denotes the vertices of $G-v$ that are adjacent to $v$. We shall write $N(v)$ when the graph $G$ appears clearly from the context. The neighborhood of the vertex $v$ in the complement of the graph $G$ will be denoted by $\bar{N}(v)$. For any subset $S$ of vertices in the graph $G$ the neighborhood of $S$ is $N(S)=\cup_{v \in S} N(v)-S$ and $N[S]=S \cup N(S)$. A clique is a subset of $V$ with the property that all the vertices are pairwise adjacent. The clique number of $G$, denoted by $\omega(G)$ is the size of the maximum clique. By $P_{n}, C_{n}, K_{n}$ we mean a chordless path on $n \geqslant 3$ vertices, the chordless cycle on $n \geqslant 3$ vertices, and the complete graph on $n \geqslant 1$ vertices. If $e=x y \in E$, we shall also write $x \sim y$, and $x \neq y$ whenever $x, y$ are not adjacent in $G$. A set $A$ is totally adjacent (non adjacent) with a set $B$ of vertices $(A \cap B=\phi)$ if $a b$ is (is not) edge, for any $a$ vertex in $A$ and any $b$ vertex in $B$; we note with $A \sim B(A \not B)$. A vertex $z \in V$ distinguishes the vertices $x, y \in V$ if $z x \in E$ and $z y \notin E$. A vertex set $M \subseteq V$ is a module if no vertex from $V-M$ distinguishes two 
vertices from $M$. A module is trivial if it is either the empty set, a one-vertex set or the entire vertex set $V$. Nontrivial modules are called homogeneous sets. A graph is prime if it contains only trivial modules. The distance $d(u, v)$ between two vertices $u$ and $v$ is the minimum of the lengths of the $u-v$ paths of $G$. The diamond is $K_{4}-e$, i.e., a four vertex clique minus one edge. The paw is the complement of $K_{1} \cup P_{3}$. The claw $\left(=K_{1,3}\right)$ is the complement of $K_{1} \cup C_{3}$. Let $F$ denote a set of graphs. A graph $G$ is $F$-free if none of its induced subgraphs is in $F$.

\section{Basic Properties}

Here we recall the notions and the results (see (Croitoru and Talmaciu, 2000), also (Talmaciu, 2002)) that are necessary in the next section. For this we define the notion of weakly component and give a characterization for the weakly decomposition of a graph.

Definition 1. Let $\mathrm{G}=(\mathrm{V}, \mathrm{E})$ be a graph. A set of vertices, $\mathrm{A}$, is called weakly set if $N_{G}(A) \neq V-A$ and the induced subgraph by A is connected. If $\mathrm{A}$ is a weakly set, maximal with respect to the inclusion, the subgraph induced by $\mathrm{A}$ is called weakly component. For simplification, the weakly component $G(A)$ will be denoted with $A$.

The name of "weakly component" is justified by the next result.

Theorem 1. Any connected and incomplete graph $G=(V, E)$ admits a weakly component A such that $G(V-A)=G(N(A))+G(\bar{N}(A))$.

Proof. Because the graph $G$ is incomplete, there are the vertices $x \neq y$, nonadjacent. We consider $A=\{x\}, B=N(x), C=\bar{N}(x)$. Clearly $y \in C$. While $(\exists b \in B$, $\exists c \in C$ such that $b c \notin E$ ) it adds $b$ to $A$ (that is $A=A \cup\{b\}$ ), it defines $B$ as being $(B-\{b\}) \cup(N(b) \cap C)$ and $C$ as being $C-(N(b) \cap C)$. Because $G(A)$ is connected and $b$ has at least a neighbour in $A$ it follows that $G(A \cup\{b\})$ is connected. Clearly $N(A \cup\{b\})=B$. Because $b c \notin E, c \in C$ and $\{c\} \not A$ it follows that $c \in C-(N(b) \cap C)$, that is $C-(N(b) \cap C) \neq \emptyset$. Because $A \subset A \cup\{b\} \subset \ldots \subset V$ and $|V|<\infty$ it follows, at last, that $N(A) \sim \bar{N}(A)$.

Theorem 2. Let $G=(V, E)$ be a connected and incomplete graph and $A \subset V$. Then $A$ is a weakly component of $G$ if and only if $G(A)$ is connected and $N(A) \sim \bar{N}(A)$.

Proof. We suppose that there is $n \in N(A)$ and $\bar{n} \in \bar{N}(A)$ such that $n \bar{n} \notin E(G)$. We consider $A^{\prime}=A \cup\{n\}$ and $N^{\prime}=(N(A)-\{n\}) \cup(N(n) \cap \bar{N}(A)) . G\left(A^{\prime}\right)$ is connected, $N\left(A^{\prime}\right)=N^{\prime}$ and $\{\bar{n}\} \subseteq V(G)-\left(A^{\prime} \cup N\left(A^{\prime}\right)\right)$. So $N\left(A^{\prime}\right) \neq V(G)-A^{\prime}$, contradicting the maximallity of $A$. Let $G(A)$ be connected and $N(A) \sim \bar{N}(A)$. We show that $G(A)$ is weakly component. We consider $A^{\prime} \supset A$, a weakly component. Because $A \not \bar{N}(A)$ and $G\left(A^{\prime}\right)$ is connected it results $\emptyset \neq A^{\prime}-A \subseteq N(A)$. We consider $n \in A^{\prime}-A$. Then $\bar{N}(A) \subseteq N(n)$. So $\bar{N}\left(A^{\prime}\right)=\emptyset$, contradicting the definition of the weakly component. 
Definition 2. A partition $(A, N(A), V-A \cup N(A))$, where $A$ is a weakly set, is called weakly decomposition of graph $G$ in relation to $A$. We call: $A$ the weakly component, $N(A)$ the minimal cutset, and $V-N(A)$ the remote set.

The next result insures the existence of a weakly decomposition in a connected and incomplete graph.

Theorem 3. If $G=(V, E)$ is a connected and incomplete graph then the set of vertices $V$ admits a weakly decomposition $(A, B, C)$ such that $G(A)$ is a weakly component and $G(V-A)=G(B)+G(C)$.

(The proof is the one given in Theorem 1.)

Theorem 2 provides an $\mathrm{O}(n+m)$ algorithm for building a weakly decomposition for an incomplete and connected graph.

Algorithm for the weakly decomposition of a graph

Input: A connected graph with at least two nonadjacent vertices, $G=(V, E)$.

Output: A partition $V=(A, N, R)$ such that $G(A)$ is connected, $N=N(A), A \not R=$ $\bar{N}(A)$.

begin

$A:=$ any set of vertices such that

$A \cup N(A) \neq V$

$N:=N(A)$

$R:=V-A \cup N(A)$

while $(\exists n \in N, \exists r \in R$ such that $n r \notin E) d o$

$A:=A \cup n$

$N:=(N-\{n\}) \cup(N(n) \cap R)$

$R:=R-(N(n) \cap R)$

end

One can observe that $[A]_{G}$ is connected, $N=N_{G}(A), R \neq \emptyset$ is an invariant of the algorithm.

Corollary 1. If $G$ is a connected graph and $(A, N, R)$ a weakly decomposition with $A$ weakly component then the following holds:

$$
\omega(G)=\max \{\omega([N])+\omega([R]), \omega([A \cup N])\} .
$$

Proof. Any clique of maximum cardinal either intersects $R$ and so it has the cardinal $\omega([N])+\omega([R])$ or it does not intersects $R$ and so it has the cardinal $\omega([A \cup N])$.

\section{The Algorithm}

In this section we establish the algorithms of recognition for the class of diamond-free graphs. 
A diamond is the graph with vertices $u, v, w, t$ and edges $u v, v t, u w, t u, w t$; a graph is called diamond-free if it has no induced subgraph isomorphic to a diamond.

In what follows, we give a characterization of a diamond-free graph.

Theorem 4. Let $G=(V, E)$ be connected with at least two nonadjacent vertices and $(A, N, R)$ a weakly decomposition with $A$ weakly component. $G$ is diamond-free if and only if we have:

(a) $G-A$ and $G-R$ are diamond-free graphs;

(b) $\forall e=a b \in E([N]): N(a) \cap N(b) \cap A=\emptyset$.

Proof. If $G$ is diamond-free then $G-A$ and $G-R$ are diamond-free. If $\exists a b \in E\left([N]_{G}\right)$ : $N(a) \cap N(b) \cap A \neq \phi$ then for $x \in N(a) \cap N(b) \cap A$ and $\forall y \in R$, the subgraph $[\{a, b, x, y\}]$ is diamond.

We suppose that (a) and (b) holds and show that $G$ is diamond-free. We suppose, however, that $\exists[D]=[\{t, u, v, w\}]$ diamond in $G$ : vw $\notin E, v t, v u, w t, w u, t u \in E$. Since $G-A$ and $G-R$ are diamond-free, $G$ connected and $A \not R$ results: $D \cap A \neq \phi$, $D \cap N \neq \phi, D \cap R \neq \phi$. If $(v \in A$ and $w \in R$ ) ( or $w \in A$ and $v \in R$ ) does not hold then, because $\{t, u\} \sim D-\{t, u\}, A \nsim R$ and $N \sim R$ results $D \cap R=\phi$ or $D \cap A=\phi$. We suppose: $v \in A$ and $w \in R$. Because $w \in R$ and $A \not R$ results $t, u \notin A$ and because $v \in A$ and $A \nsim R$ results $t, u \notin R$. So: $u, t \in N ; v \in A$ and $w \in R$. But then $t u \in E([N])$ for which $N(u) \cap N(t) \cap A \neq \phi$, contradicting (a).

The above result leads to the following recognition algorithm.

Input: $G=(V, E)$ a connected graph with at least two nonadjacent vertices.

Output: An answer to the question: Is $G$ a diamond-free graph?

1. $\{L=G ; / / L$ a list of graphs

2. while $(L \neq \emptyset)$

\{ extract an element $H$ from $L$;

find a weakly decomposition $(A, N, R)$ for $H$;

if $\left(\exists a b \in E([N])_{G}\right.$ such that $\left.N(a) \cap N(b) \cap A \neq \emptyset\right)$ then

Return: $G$ is not diamond-free;

else introduce in $L$ the connected components of $G-R, G-A$ incomplete

3. Return: $G$ is diamond-free \}

\}

Although the complexity of this algorithm - in the worst case - is not better than those of the most efficient, known algorithms, its behaviour is superior in many implementations. This is because the above algorithm takes into account the combinatorial structure of the graph, by means of the decomposition that has been used.

\section{References}

Berge, C. (1985). Graphs. Nort-Holland, Amsterdam.

Brandstadt, A. (2004). ( $P_{5}$, diamond)-free graphs revited: structure and linear time optimization. Discrete Applied Math., 138, 13-27. 
Brandstadt, A., T.C. Hoang and J.-M. Vanherpe (2002). On minimal prime extensions of a four-vertex graph in a prime graph. Manuscript.

Brandstadt, A., H.-O. Le, V.V. Lozin (2003). New graph classes of bounded clique-width II. Rutcor Research Report 34-2003.

Croitoru, C., and M.Talmaciu (2000). A new graph search algorithm and some applications. Presented at ROSYCS 2000, Univ. Al.I.Cuza Iaşi.

Kloks, T., D. Kratsch and H. Muller. Finding and counting small induced subgraphs efficiently. Manuscript.

Talmaciu, M. (2002). Decomposition Problems in the Graph Theory with Applications in Combinatorial Optimization. Ph. D. University Al. I. Cuza Iasi, Romania.

M. Talmaciu is an associate professor, $\mathrm{PhD}$, a head of the Department of Mathematics and Computer Science of the University of Bacău, Romania. Main research interests are graph theory and numerical analysis.

E. Nechita is an associate professor, $\mathrm{PhD}$, at the Department of Mathematics and Computer Science of the University of Bacău, Romania. Main research interests are natural computing and probability theory.

\section{Rombụ neturinčiu grafų atpažinimo algoritmas}

\section{Mihai TALMACIU, Elena NECHITA}

Straipsnyje pateikiamas silpnos dekompozicijos apibrèžimas bei būtinos ir pakankamos sąlygos, kad būtụ ịmanoma tokia grafo dekompozicija. Ivedamas atpažinimo algoritmas rombụ neturinčių grafų atpažinimui, kuris išlaiko kombinatorinę grafo struktūrą naudodamas minètą dekompoziciją. Algoritmas taip pat suteikia galimybę lengvai nustatyti rombo neturinčio grafo grupès numerị. 